\title{
Defects in growth and bone metabolism in klotho mutant mice are resistant to $\mathrm{GH}$ treatment
}

\author{
K Kashimada, T Yamashita, K Tsuji, A Nifuji, S Mizutani ${ }^{1}$, \\ Y Nabeshima ${ }^{2,3}$ and $M$ Noda \\ Department of Molecular Pharmacology, Medical Research Institute, Tokyo Medical and Dental University, Kanda Surugadai 2-3-10, Chiyoda-ku, \\ Tokyo 101-0062, Japan \\ ${ }^{1}$ Department of Human Ontogeny and Childhood Development, Tokyo Medical and Dental University, Tokyo, Japan \\ ${ }^{2}$ Department of Pathology and Tumor Biology, Graduate School of Medicine, Kyoto University, Kyoto, Japan \\ ${ }^{3}$ Core Research for Evolutional Science and Technology, Japan Science and Technology Corp., Saitama, Japan \\ (Requests for offprints should be addressed to M Noda; Email: noda.mph@mri.tmd.ac.jp)
}

\begin{abstract}
Klotho mutant $(\mathrm{kl} / \mathrm{kl})$ mice exhibit growth retardation after weaning, and previous electron microscopic examination of GH-producing cells in pituitary glands revealed a reduction in $\mathrm{GH}$ granules. However, it has not been known whether growth retardation in klotho mutant mice is related to the loss of $\mathrm{GH}$ function. We therefore examined whether treatment with GH could rescue the retardation of growth. At the end of 3 weeks of treatment with human GH, the body weight of wild-type (WT) mice was increased. In contrast, body weight was not increased in klotho mutant mice even after the treatment with human GH. Another feature of klotho mutant mice is the presence of osteopetrosis in the epiphyses of long bones
\end{abstract}

and vertebrae. Treatment with human GH increased trabecular bone volume in the epiphyseal region of WT tibiae. Interestingly, increase in trabecular bone volume by $\mathrm{GH}$ treatment was also observed in klotho mutant mice and, therefore, the phenotype of high bone volume in the klotho mice was further enhanced. These findings indicate that a GH receptor system in cancellous bones could operate in mutant mice. Thus, growth retardation in the klotho mutant mice is resistant against GH treatment even when these mice respond to $\mathrm{GH}$ treatment in terms of cancellous bone volume.

Journal of Endocrinology (2002) 174, 403-410

\section{Introduction}

Klotho mutant mice were discovered based on their severe growth retardation, short lives and their phenotypes including emphysema, atherosclerosis, gonadal dysplasia, ectopic calcification, ataxia, skin atrophy, defects in hearing, thymic atrophy and defects in B-cell differentiation (Kuro-o et al. 1997, Lyons 1997, Roush 1997). Some of these manifestations resemble aging-associated phenomena. Klotho gene encodes a putative membrane protein (Matsumura et al. 1998, Shiraki-Iida et al. 1998) with two $\beta$-glucosidase like domains, called KL1 and KL2. However, its precise function has not yet been identified (Aizawa et al. 1998, Ohyama et al. 1998, Saito et al. 1998, 1999, Kato et al. 2000, Shapiro 2000, Suga et al. 2000).

The bones in klotho mutant mice are normal until weaning, but due to the growth retardation after weaning, their long bones are shorter than those in age-matched wildtype (WT) mice (Kuro-o et al. 1997). About 4 weeks after birth, cancellous bones in the metaphyseal regions of long bones as well as vertebrae in klotho mutant mice become dense, based on X-ray analysis (Yamashita et al. 1998). Micro CT examination revealed that bone volume fraction in these regions in klotho mice is higher than that in the corresponding regions in the bones of WT mice (Kawaguchi et al. 1999, Yamashita et al. 2000a). A bone marrow ablation study indicated that new bone formation after bone marrow ablation in klotho mutant mice was observed within one week, similar to that seen in WT mice (Yamashita et al. 2000b). In WT mice, newly formed trabecular bones were resorbed within two weeks after bone marrow ablation. However, no such bone resorption was observed in klotho mutant mice, indicating the presence of a defect in bone resorption (Yamashita et al. 2000b). The osteopetrotic phenotype in bone is always observed to be associated with the onset of growth retardation and other phenotypes in klotho mutant mice.

The homozygous klotho mutant mice grow normally until weaning; however, their growth starts to retard 2-3 weeks after birth. Although this growth retardation appears similar to the phenotype in mice with growth hormone (GH) deficiency (Van Buul \& Van den Brande 

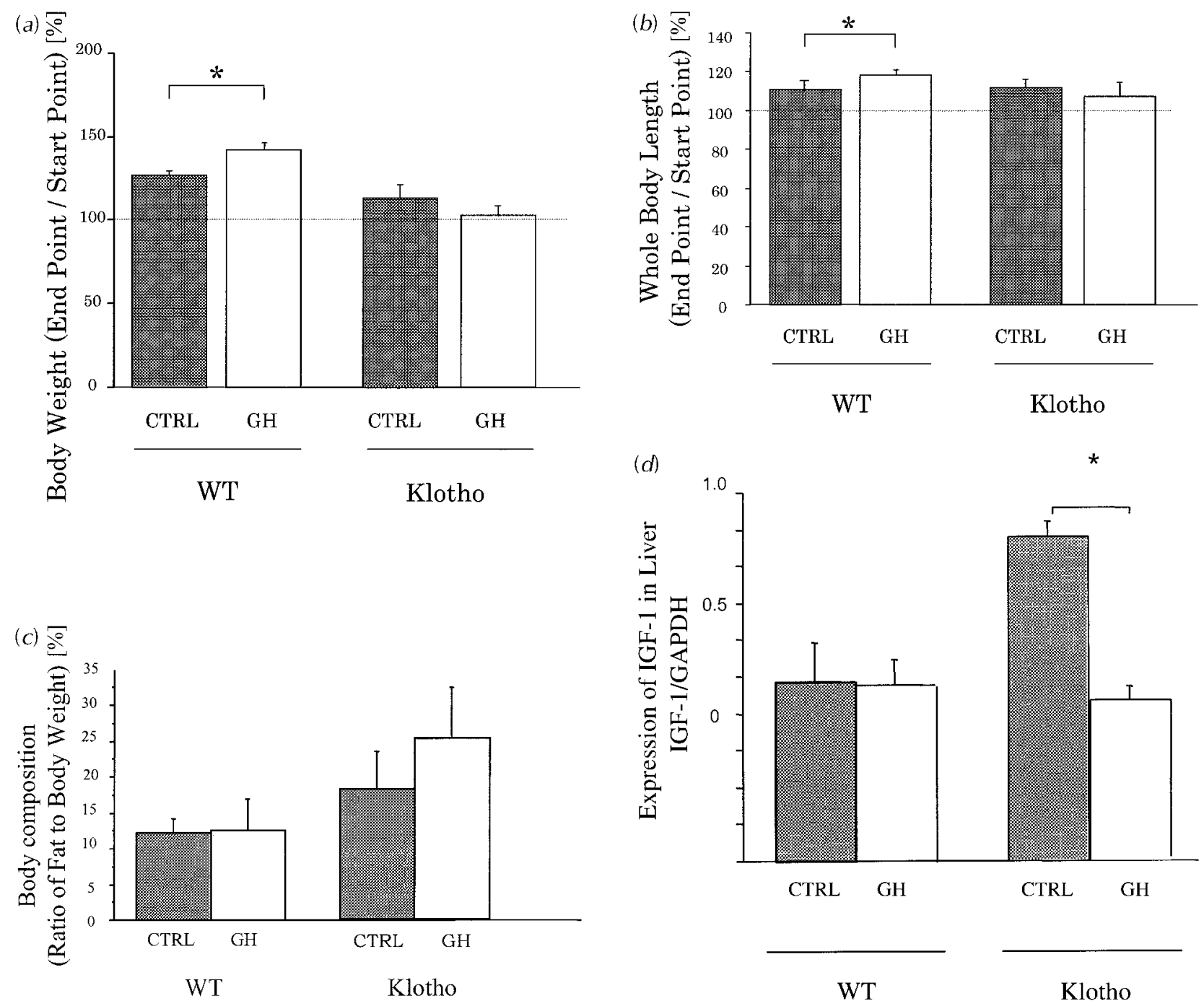

Figure 1 Growth of mice in response to human GH treatment. (a) The body weight of mice after treatment with human GH. The body weight was measured at the start point and the end point of the experiments and the ratios of the body weight were obtained. (b) The whole body length of mice. The body length was measured at the start point and end point of the experiments on the soft X-ray films, and the ratio of the body length was obtained. (c) The body composition of mice. The ratios of fat/body weight were determined using DEXA. (d) IGF-I mRNA levels in the liver of mice. RT-PCR was conducted using liver RNA prepared $24 \mathrm{~h}$ after GH injection. IGF-I mRNA data were normalized against GAPDH mRNA levels. Mice were prepared as described in Materials and Methods and were injected with GH or saline (CTRL) for 3 weeks. Asterisks indicate significant difference $(P<0 \cdot 05)$. Means \pm S.D.

1978), it has not been known whether any defects related to $\mathrm{GH}$ are involved in the retardation of the growth of klotho mutant mice except for the report that electron microscopic examination showed reduction in the secretory granules in GH-secreting cells in the pituitary glands (Kuro-o et al. 1997). Therefore, we examined whether treatment with GH could rescue retardation of growth in klotho mutant mice. We also examined whether GH treatment would affect osteopetrotic phenotype in the klotho mutant mice.

\section{Materials and Methods}

Human GH was a kind gift from Eli Lilly Co. Klotho mutant mice and WT mice were obtained by crossing heterozygote mice $(\mathrm{kl} /+)$ with a TA20 strain background with mutation in the klotho gene locus $(\mathrm{C} 3 \mathrm{H} / \mathrm{J}$ and C57BL/6J mixed background), as described previously. WT and klotho mutant mice were used for this study. Four-week-old mice were s.c. injected with $3 \mu \mathrm{g} / \mathrm{g}$ of recombinant human GH per day for 3 weeks (one 
CTRL

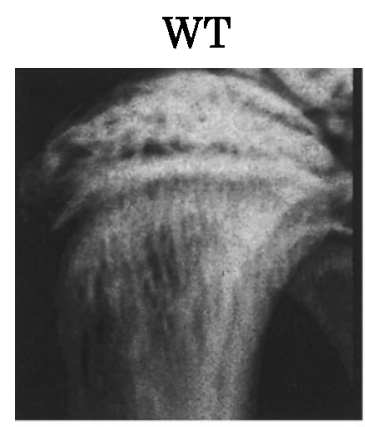

CTRL

Klotho

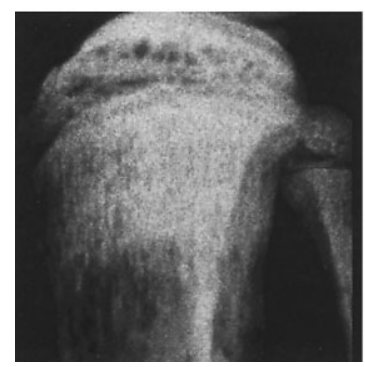

GH

WT

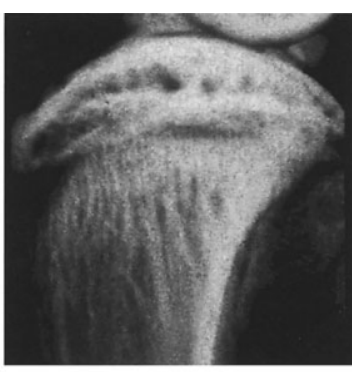

GH

\section{Klotho}

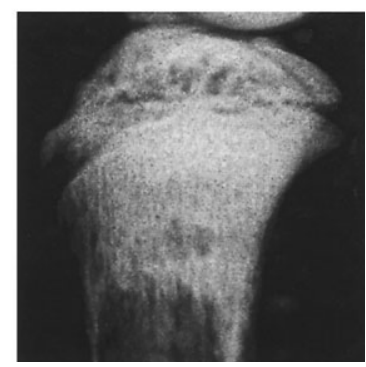

Figure 2 X-ray pictures of the epiphyseal regions of tibiae. At the end of 3 weeks of treatment with $\mathrm{GH}$ or saline (CTRL), metaphyseal regions were subjected to X-ray examination. High radiopacity was observed in klotho mutant mice but not in WT mice and osteopetrotic changes were observed in klotho mutant mice regardless of the $\mathrm{GH}$ treatment.

injection per day, 5 days per week). This dosage of the GH was set according to the previously published data (Van Buul \& Van den Brande 1978). As control, half of the mice in each of the groups were injected with saline. Body weight was recorded every day.

\section{Body composition}

We estimated the weight of fatty tissue in whole body by using a dual-energy X-ray absorptiometry (DEXA) apparatus (Pixi-MUS; Lunar, WI, USA).

\section{$X$-ray and bone mineral density $(B M D)$ analyses}

X-ray pictures of tibiae were taken at $20 \mathrm{kV}$ and $0.8 \mathrm{~mA}$ for 2 min after killing. BMD was measured using a small animal version of the Pixi-MUS DEXA apparatus. BMD was measured in the epiphyses of tibiae at their proximal ends (one-fifth of the total length) and in the diaphyses of tibiae (three-fifths of the total length).
Three dimensional (3D) micro-computed tomography (CT) analysis

The 3D trabecular bone analysis was conducted using a desk-top 3D micro-CT analysis system (Micro-CT20; Scanco Medical, Zurich, Switzerland), as described previously (Yamashita et al. 2000a). In brief, the bone samples were fixed with $70 \%$ ethanol after killing and the measurements were conducted in a globular region with a diameter of $0.80 \mathrm{~mm}$ in the epiphyses adjacent to $(0.36 \mathrm{~mm}$ away from $)$ the growth plates. Thresholds for measurement were set at 275 for $3 \mathrm{D}$ micro-CT analysis.

\section{$R N A$ preparation and semi-quantitative $R T-P C R$}

Animals (three mice per group, total of 12 mice) were killed $24 \mathrm{~h}$ after the last $\mathrm{GH}$ or saline injection, and livers were collected. Total RNA was isolated according to the acid-guanidium-phenol-chloroform (AGPC) method. The first-strand DNA synthesis was conducted as follows. Aliquots of RNA were incubated at $65^{\circ} \mathrm{C}$ for $10 \mathrm{~min}$ using oligo $\mathrm{dT}$ primer (Roche), followed by incubation at $37^{\circ} \mathrm{C}$ for $60 \mathrm{~min}$ in the presence of RNAase inhibitor (Promega) and MMLV RT polymerase (Gibco Life Technologies). One microgram of cDNA was amplified using primers as described previously (Oomizu et al. 1998) for 24 cycles. The cycle number of PCR amplification was set to obtain band intensities within a linear range. Ethidium bromide stained bands were quantitated using an image analyzer, Bio-1D system (Vilber Lourmat, Marne, France). As a control, glyceraldehyde-3-phosphate dehydrogenase (GAPDH) mRNA levels were used for normalization. Data were expressed as ratios of insulin-like growth factor-I (IGF-I)/ GAPDH.

\section{Histomorphometric analysis}

Femora were fixed in $4 \%$ paraformaldehyde/PBS, and decalcified with $10 \%$ formic acid for 3 days. The samples were embedded in paraffin and $5 \mu \mathrm{m}$ thickness sections were made and stained with toluidine blue.

\section{Statistical analysis}

All data are expressed as means \pm S.D. Statistical significance of the difference in the values was evaluated by the Mann-Whitney U-test. Difference with $P$ values less than 0.05 were regarded as statistically significant.

\section{Results}

By the end of the 3 week experimental period (mice were 7 weeks old at the end of the experiments), the body weight of WT mice injected with saline alone was 

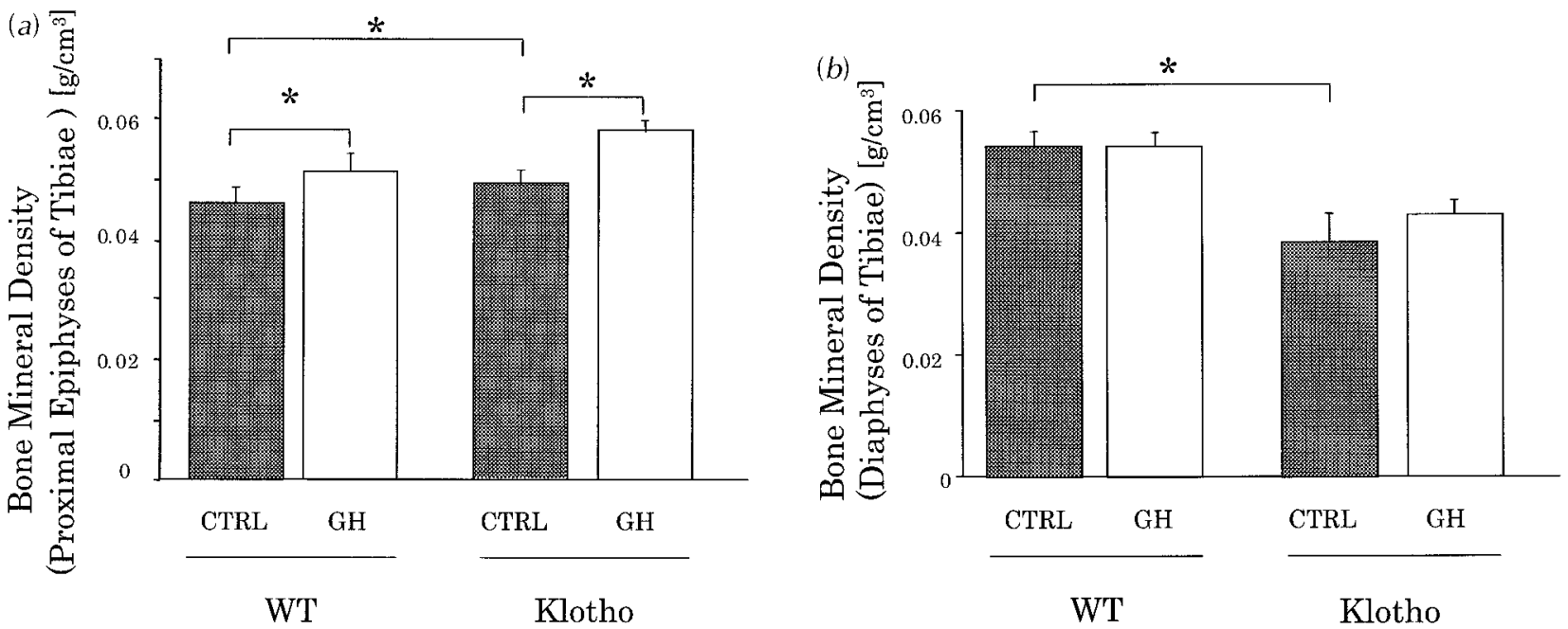

Figure $3 \mathrm{BMD}$ based on DEXA. At the end of 3 weeks of treatment with GH or saline (CTRL), tibiae were subjected to DEXA as described in Materials and Methods. (a) BMD in the proximal epiphyseal regions of tibiae. (b) BMD in the diaphyses of tibiae. Asterisks indicate significant differences $(P<0 \cdot 05)$. Means \pm S.D.

increased by $28 \cdot 6 \pm 2 \cdot 3 \%$. As reported previously (Van Buul \& Van den Brande 1978), treatment with human $\mathrm{GH}$ enhanced the increase in the body weight of WT mice by about $40 \%$ during the 3 weeks $(P<0 \cdot 05$, Fig. 1 a). In control klotho mutant mice, which were injected with saline alone, body weight increment was $13.9 \pm 5 \cdot 3 \%$ and no statistically significant difference between the body weights at the start and end point of the 3 week period was observed. Furthermore, in contrast to the WT mice, the body weight increase after the 3 week treatment with GH was only $4 \cdot 0 \pm 5 \cdot 9 \%$ and no statistical difference was observed between the body weights of $\mathrm{GH}$ - and salinetreated klotho mice (Fig. 1a). Thus, GH did not rescue the growth retardation in klotho mice.

The whole body length, measured on X-ray from nose to sacrum, was increased by $10 \cdot 4 \pm 3 \cdot 3 \%$ in the salinetreated control group and by $17 \cdot 0 \pm 2 \cdot 6 \%$ in the GHtreated group $(P<0 \cdot 05)$ (Fig. 1b) in WT. The body length in klotho mutant mice was increased by $12 \cdot 3 \pm 3.2 \%$ in the control group and by $7 \cdot 6 \pm 6 \cdot 3 \%$ in the GH-treated group (Fig. 1b). Thus, no GH effect on the whole body length was observed in the mutant mice.

We also examined whether no change in the body weight in GH-treated klotho mutant mice was associated with change in body composition. GH treatment did not significantly alter body composition regarding fat to tissue ratio in either klotho mutant mice or WT (Fig. 1c).
We further examined IGF-I gene expression levels by RT-PCR using livers of the mice. Contrary to our prediction, expression levels of IGF-I mRNA in liver in klotho mice were elevated compared with the WT (Fig. 1d). Furthermore, IGF-I mRNA levels were similar to the control $24 \mathrm{~h}$ after the last GH injection in WT mice. In contrast, GH treatment normalized the levels of IGF-I mRNA to the levels similar to the saline-injected group $24 \mathrm{~h}$ after the last injection in klotho mutant mice.

$\mathrm{X}$-ray analysis of the proximal ends of the tibiae of klotho mutant mice taken at the end of the 3 week period indicated the presence of radiopacity in the epiphyses of long bones (Fig. 2) and vertebrae (data not shown) in the saline-injected group as reported previously (Kuro-o et al. 1997, Yamashita et al. 1998). Such high radiopacity was still observed in the klotho mutant mice after 3 weeks of treatment with GH (Fig. 2).

BMD in the proximal epiphyses of the tibiae (proximal one-fifth of the total length) was increased significantly by the GH treatment in WT mice (Fig. 3a, $P<0.05$ ). Basal levels of BMD in saline-injected klotho mutant mice were higher than those in saline-injected WT (Fig. 3a, $P<0 \cdot 05$ ). $\mathrm{GH}$ treatment also enhanced the level of BMD in the proximal regions of the tibiae in klotho mutant mice (Fig. $3 \mathrm{a}, P<0 \cdot 05)$. These observations indicated that the response to $\mathrm{GH}$ at the levels of cancellous bone tissues was not impaired in klotho mice. In contrast, BMD of the

Figure 4 3D micro-CT analysis of trabecular bone of tibiae. (a) 3D images of micro-CT analysis. Proximal ends of tibiae are shown. (b-h) Morphological analysis of trabecular bone. The measurements were conducted as described in Materials and Methods, and all parameters were analyzed by software based on an IPL package in the micro-CT apparatus. (b) Bone volume/tissue volume. (c) Trabecular number. (d) Trabecular thickness. (e) Bone surface. (f) Bone surface/bone volume. (g) Trabecular separation. (h) Anisotropy. Asterisks indicate significant difference $(P<0 \cdot 05)$. CTRL $=$ control saline-treated mice. Means \pm S.D. 
(a) $\mathrm{WT}$
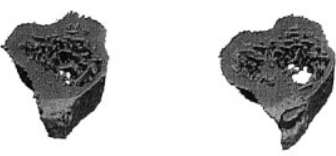

CTRL

$\mathrm{GH}$

Klotho
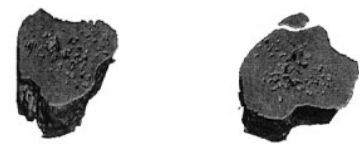

CTRL

GH

(c)

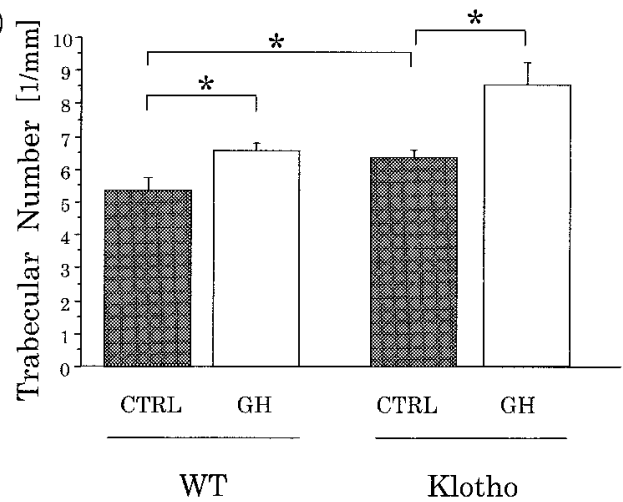

(e)
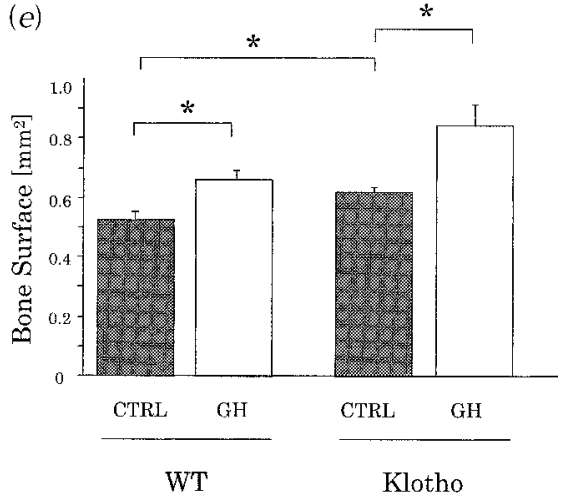

(g)

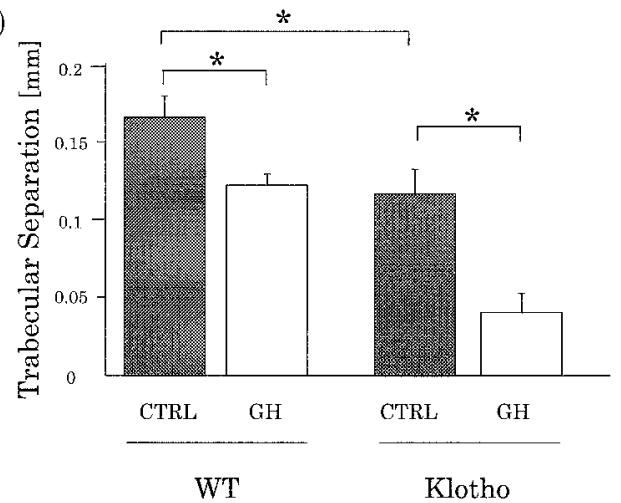

www.endocrinology.org (b)

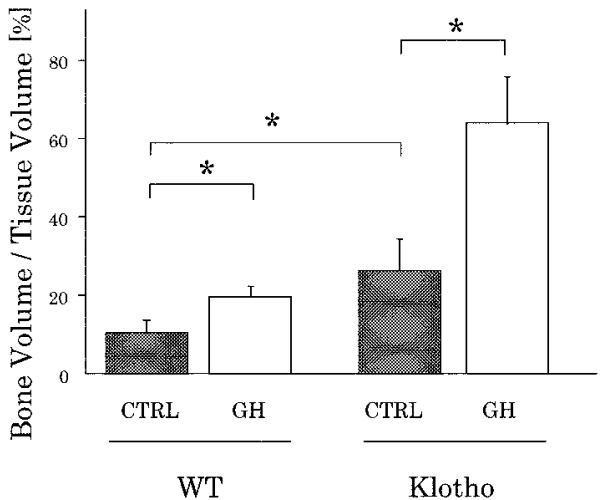

(d)
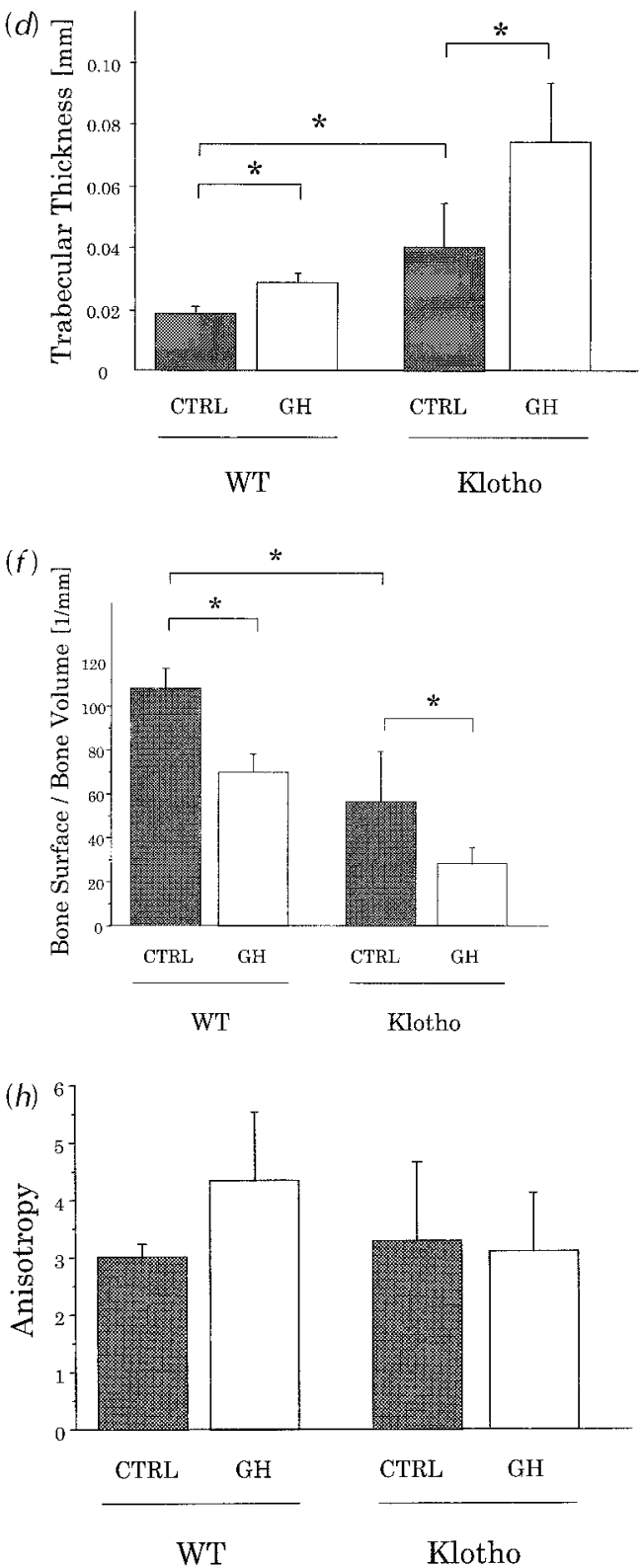

Journal of Endocrinology (2002) 174, 403-410

Downloaded from Bioscientifica.com at 04/26/2023 09:10:08AM 
WT
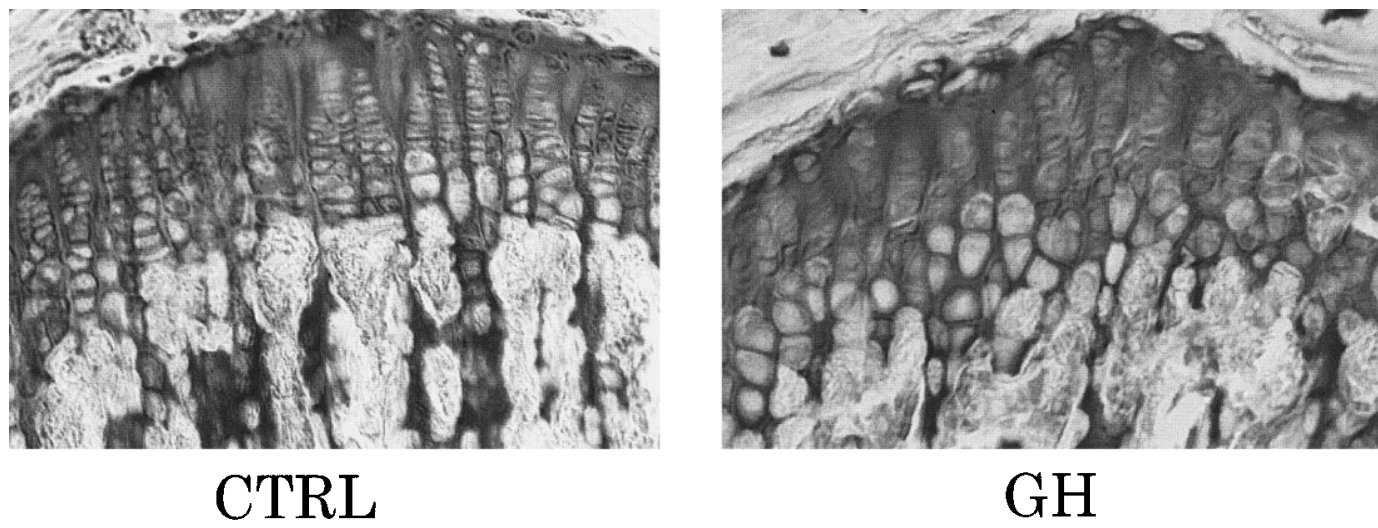

\section{Klotho}

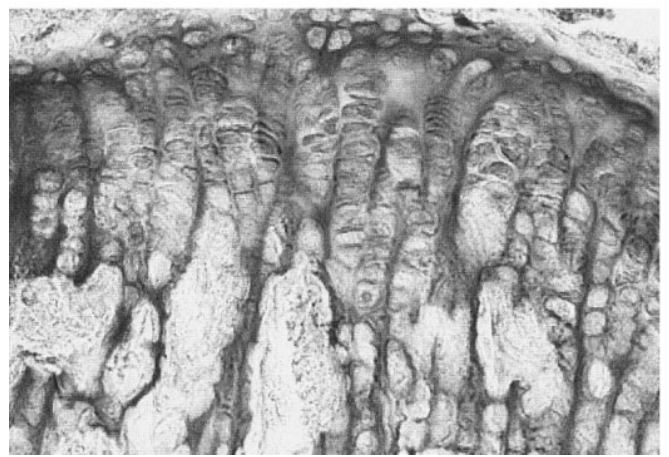

CTRL

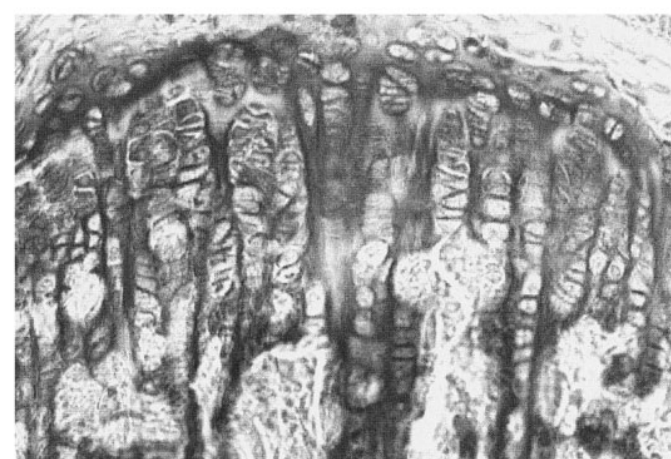

GH

Figure 5 Growth plate of the femora. WT and klotho mutant mice were treated with GH or saline (CTRL). Irregularity of chondrocyte columns in klotho mutant mice was not rescued by a 3 week $\mathrm{GH}$ treatment.

diaphyseal cortical bone (middle three-fifths of the total length) was not increased by the GH treatment in both groups, i.e. WT mice and klotho mutant mice. Therefore, osteoporotic changes in the cortical bone known to exist in klotho mutant mice were not improved by the $\mathrm{GH}$ treatment (Fig. 3b).

To investigate the structural aspects of the response of trabecular bone to $\mathrm{GH}$ treatment more precisely, we examined the trabecular bone in the epiphyses of tibiae by using a 3D micro-CT analysis system (Fig. 4a). Trabecular bone volume/tissue volume (BV/TV), trabecular thickness, trabecular bone surface and trabecular number were significantly increased by GH treatment in WT mice (Fig. $4 \mathrm{~b}-\mathrm{e})$ and similar $\mathrm{GH}$ effects were observed in klotho mutant mice (Fig. 4b-e). Trabecular separation and bone surface ratio to bone volume were significantly decreased by GH treatment in both groups (Fig. 4f and g). Anisotropy, which indicates directions of the trabecular axis, was not significantly altered by GH treatment (Fig. $4 \mathrm{~h})$.

In WT mice, chondrocytes in the growth plate formed columns along the longitudinal axis of the femora and such chondrocyte columns appeared to be elongated after GH treatment (Fig. 5). In klotho mutant mice, chondrocytes did not clearly form columns, and the size and the shape of chondrocyte columns were irregular. These phenotypes of chondrocytes in klotho mutant mice were not rescued by the GH treatment (Fig. 5).

\section{Discussion}

Growth retardation is one of the representative phenotypes in klotho mutant mice (Kuro-o et al. 1997). In these mice, electron microscopic examination indicated that the number of vesicles in the GH-producing cells in the pituitary gland was reduced (Kuro-o et al. 1997), suggesting that the growth retardation in klotho mutant mice could be in part due to GH deficiency (Kuro-o et al. 1997). We therefore examined directly the effect of exogenous $\mathrm{GH}$ on the growth retardation in klotho mutant mice and found that the mechanism responsible for the growth retardation is independent from GH function.

The increase in BMD in the epiphyses after GH treatment of klotho mutant mice suggests that at least in the epiphyseal cancellous bone regions, the GH pathway 
appears to affect the metabolism of klotho mutant mice similarly to the WT. Therefore, klotho gene mutation is potent enough to override the growth-promoting effects of $\mathrm{GH}$ used in our experiment. A recent report on the abnormality in the energy homeostasis in the klotho mutant mice (Mori et al. 2000, Utsugi et al. 2000) may also suggest such a defect independent of the GH pathway in klotho mutant mice. In this report, glucose tolerance and insulin sensitivity in klotho mice are shown to be increased compared with those in WT mice based on i.p. glucose and insulin tolerance tests (Mori et al. 2000).

Lack of changes in the body weight of klotho mice may not mean that there is no tissue response to GH therapy. For example, GH may be increasing fat-free mass but decreasing fat mass to the same extent with no net effect on body weight. However, in our experiments the body composition was not significantly altered in either klotho mutant mice or WT mice by GH treatment.

In addition to body weight gain, we also examined the effect of $\mathrm{GH}$ on the bone phenotypes in these mice (Yamashita et al. 1998, 2000a,b, Kawaguchi et al. 1999). GH treatment significantly increased trabecular bone volume, but not cortical bone in WT mice. This site-specific effect of $\mathrm{GH}$ was also observed in bovine $\mathrm{GH}$ transgenic mice (Graichen et al. 1999). These site-specificities were maintained in klotho mutant mice. Klotho mutant mice show osteopetrosis in their metaphyseal regions about 4 weeks after birth (Yamashita et al. 1998). This has been seen in association with high levels of osteoprotegerin expression in the bone marrow (Yamashita et al. 2000b). The osteopetrosis in the metaphyses is one of the early phenotypic changes in klotho mutant mice. As mentioned above, GH treatment increased trabecular bone volume and BMD in the epiphyseal regions of tibiae in klotho mice indicating that the $\mathrm{GH}$ receptor and down-stream signaling system could be operating. However, GH did not improve the levels of osteopetrosis. Therefore, in klotho mutant mice, there would be a defect in body systems other than GH/IGF-I axis stimulation.

Against our prediction, IGF-I mRNA in the liver in klotho mice was elevated. Furthermore, GH treatment normalized the IGF-I mRNA levels in the liver in klotho mice. This observation cannot be simply explained based on the currently accepted understandings on the GH/ IGF-I system. It appears that an as yet unidentified novel system might enhance IGF-I mRNA expression in the liver in klotho mice and that such a system might also normalize the IGF-I mRNA levels upon GH treatment. Even if this is the case, whether such IGF-I mRNA elevation is a compensatory response related to growth retardation in klotho mice or alternatively is totally independent from growth abnormality in these mice is still to be elucidated. IGF-I mRNA levels were similar to control $24 \mathrm{~h}$ after the last GH injection in WT mice. This may indicate that the effects of GH injection on IGF-I mRNA levels could return to base line within $24 \mathrm{~h}$ in WT mice.
In conclusion, growth retardation in klotho mutant mice is resistant to $\mathrm{GH}$ treatment even though this hormone can act on trabecular bone in these mice.

\section{Acknowledgements}

This research was supported by the grants-in-aid received from the Japanese Ministry of Education (14207056, 14034214, 14028022, 12557123, 13045011, 13216034), Grants from NASDA, Japan Society for Promotion of Science (Research for the Future Program, Genome Science) and Tokyo Biochemistry Research Foundation.

\section{References}

Aizawa H, Saito Y, Nakamura T, Inoue M, Imanari T, Ohyama Y, Matsumura Y, Masuda H, Oba S, Mise N, Kimura K, Hasegawa A, Kurabayashi M, Kuro-o M, Nabeshima Y \& Nagai R 1998 Downregulation of the klotho gene in the kidney under sustained circulatory stress in rats. Biochemical and Biophysical Research Communications 249 865-871.

Graichen H, Lochmuller E, Wolf E, Langkabel B, Stammberger T, Haubner M, Renner-Muller I, Englmeier K \& Eckstein F 1999 A non-destructive technique for 3-D micro structural phenotypic characterisation of bones in genetically altered mice: preliminary data in growth hormone transgenic animals and normal controls. Anatomy and Embryology 199 239-248.

Kato Y, Arakawa E, Kinoshita S, Shirai A, Furuya A, Yamano K, Nakamura K, Iida A, Anazawa H, Koh N, Iwano A, Imura A, Fujimori T, Kuro-o M, Hanai N, Takeshige K \& Nabeshima Y 2000 Establishment of the anti-klotho monoclonal antibodies and detection of klotho protein in kidneys. Biochemical and Biophysical Research Communications 267 597-602.

Kawaguchi H, Manabe N, Miyaura C, Chikuda H, Nakamura K \& Kuro-o M 1999 Independent impairment of osteoblast and osteoclast differentiation in klotho mouse exhibiting low-turnover osteopenia. Journal of Clinical Investigation 104 229-237.

Kuro-o M, Matsumura Y, Aizawa H, Kawaguchi H, Suga T, Utsugi T, Ohyama, Y Kurabayashi M, Kaname T, Kume E, Iwasaki HA, Shiraki-Iida T, Nishikawa S, Nagai R \& Nabeshima YI 1997 Mutation of the mouse klotho gene leads to a syndrome resembling ageing. Nature 390 45-51.

Lyons EJ 1997 Evolutionary biology. Sex and synergism. Nature 390 19-21.

Matsumura Y, Aizawa H, Shiraki-Iida T, Nagai R, Kuro-o M \& Nabeshima Y 1998 Identification of the human klotho gene and its two transcripts encoding membrane and secreted klotho protein. Biochemical and Biophysical Research Communications 242 626-630.

Mori K, Yahata K, Mukoyama M, Suganami T, Makino H, Nagae T, Masuzaki H, Ogawa Y, Sugawara A, Nabeshima Y \& Nakao K 2000 Disruption of klotho gene causes an abnormal energy homeostasis in mice. Biochemical and Biophysical Research Communications 278 665-670.

Ohyama Y, Kurabayashi M, Masuda H, Nakamura T, Aihara Y, Kaname T, Suga T, Arai M, Aizawa H, Matsumura Y, Kuro-o M, Nabeshima Y \& Nagail R 1998 Molecular cloning of rat klotho cDNA: markedly decreased expression of klotho by acute inflammatory stress. Biochemical and Biophysical Research Communications $251920-925$.

Oomizu S, Takeuchi S \& Takahashi S 1998 Stimulatory effect of insulin-like growth factor I on proliferation of mouse pituitary cells in serum-free culture. Journal of Endocrinology 157 53-62. 
Roush W 1997 Fast-forward aging in a mutant mouse? Science $\mathbf{2 7 8}$ 1013.

Saito Y, Yamagishi T, Nakamura T, Ohyama Y, Aizawa H, Suga T, Matsumura Y, Masuda H, Kurabayashi M, Kuro-o M, Nabeshima Y \& Nagai R 1998 Klotho protein protects against endothelial dysfunction. Biochemical and Biophysical Research Communications 248 324-329.

Saito Y, Kuroo M, Nabeshima Y \& Nagai R 1999 The protective role of klotho gene on vascular endothelium. Japanese Journal of Clinical Medicine 57 514-518.

Shapiro SD 2000 Animal models for chronic obstructive pulmonary disease: age of klotho and marlboro mice. American Journal of Respiratory Cell and Molecular Biology 22 4-7.

Shiraki-Iida T, Aizawa H, Matsumura Y, Sekine S, Iida A, Anazawa H, Nagai R, Kuro-o M \& Nabeshima Y 1998 Structure of the mouse klotho gene and its two transcripts encoding membrane and secreted protein. FEBS letters 424 6-10.

Suga T, Kurabayashi M, Sando Y, Ohyama Y, Maeno T, Maeno Y, Aizawa H, Matsumura Y, Kuwaki T, Kuro-o M, Nabeshima Y \& Nagai R 2000 Disruption of the klotho gene causes pulmonary emphysema in mice. Defect in maintenance of pulmonary integrity during postnatal life. American Journal of Respiratory Cell and Molecular Biology 22 26-33.

Utusgi T, Ohno T, Ohyama Y, Uchiyama T, Saito Y, Matsuyama Y, Aizawa H, Itoh H, Kurabayashi M, Kawazu S, Tomono S, Oka Y,
Suga T, Kuro-o M, Nabeshima Y \& Nagai R 2000 Decreased insulin production and increased insulin sensitivity in the klotho mutant mouse, a novel animal model for human aging. Metabolism: Clinical and Experimental 49 1118-1123

Van Buul S \& Van den Brande J 1978 The Snell dwarfmouse. I. General growth pattern, before and during growth hormone and thyroxine therapy. Acta Endocrinologica 89 632-645.

Yamashita T, Nifuji A, Furuya K, Nabeshima Y \& Noda M 1998 Elongation of the epiphyseal trabecular bone in transgenic mice carrying a klotho gene locus mutation that leads to a syndrome resembling aging. Journal of Endocrinology 159 1-8.

Yamashita T, Nabeshima Y \& Noda M 2000a High-resolution micro-computed tomography analyses of the abnormal trabecular bone structures in klotho gene mutant mice. Journal of Endocrinology $164239-245$

Yamashita T, Yoshitake H, Tsuji K, Kawaguchi N, Nabeshima Y \& Noda M $2000 b$ Retardation in bone resorption after bone marrow ablation in klotho mutant mice. Endocrinology 141 438-445.

Received 10 April 2002

Accepted 15 May 2002 